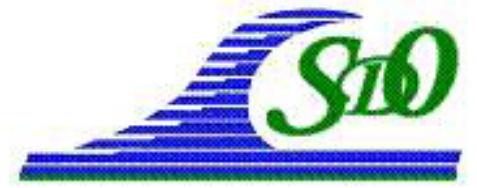

Xìmes Journées Nationales Génie Côtier - Génie Civil

Les Sables d'Olonne, 22-25 juin 2010

DOI:10.5150/jngcgc.2010.093-L C Editions Paralia CFL

disponible en ligne - http://www.paralia.fr - available online

\title{
Déshydratation mécanisée in situ de sédiments fraîchement dragués ou mis en dépôts : le projet SEDIGATE ${ }^{\circledR}$ I
}

\author{
Daniel LEVACHER ${ }^{1}$, Philippe DHERVILLY ${ }^{2}$
}

1. Université de Caen, Faculté des Sciences, UMR 6143 CNRS - M2C, Esplanade de la Paix, 14032 Caen, France.

daniel.levacher@unicaen.fr

2. ARD Normandie, 14000 Hérouville Saint-Clair, France.

pdhervilly@ard-normandie.fr

\section{Résumé :}

La gestion à terre des sédiments de dragage pose problème et reste un défi majeur à relever pour la prochaine décennie. Il s'agit d'une part (i) de valoriser les volumes importants de sédiments sains régulièrement dragués (source renouvelable) et immergés en mer et d'autre part, (ii) de recycler des volumes réduits de sédiments pollués. Pour l'une de ces actions, il s'agit d'une solution alternative à la pratique du clapage qui permet d'éviter d'impacter les fonds marins. Pour l'autre, elle conduit à l'élimination progressive des stocks de sédiments pollués. Mais la gestion de ces volumes conséquents nécessite une valorisation ou un recyclage à terre à l'échelle industrielle. C'est l'objet du projet SEDIGATE I qui est de conditionner ces sédiments avant de procéder à leur stabilisation/solidification. L'article proposé concerne tout d'abord la définition du projet SEDIGATE I, les objectifs attendus, et les moyens mis en œuvre. Ce projet s'inscrit dans la gestion à terre des sédiments dont l'acronyme est tiré : SEDIments - Gestion Á TErre. L'objectif du programme numéro I est de réaliser une déshydratation mécanisée des sédiments fraîchement dragués ou en sortie de drague. Ceci passe par la mise au point d'unités de traitement permettant de déshydrater, de dessabler, de trier et de proposer un sédiment à forte siccité prêt à être traité sur place ou à être transporté vers différents postes de traitement de stabilisation et de solidification. Ce programme repose sur l'utilisation d'une machine à déshydrater les sédiments et a pour objet l'étude des performances d'une machine à déshydrater les sédiments. Cette machine a été construite à partir d'un procédé breveté, elle assure un tri et fournit des sédiments déshydratés pour une siccité voisine de $25 \%$ à $30 \%$. La première phase consiste à la mise en œuvre de la machine sur un site portuaire, d'en définir les performances et les limites d'utilisation. L'article après la présentation du programme fait état des éléments composant la machine à déshydrater les sédiments dont la première application concerne des sédiments de Seine.

\section{Mots-clés :}

Sédiments - Déshydratation - SEDIGATE - Dragage - Séparation solide/liquide Valorisation - Développement durable - Unité mobile de conditionnement 


\section{Introduction}

Les gestionnaires de ports, du grand port maritime au petit port de plaisance, et des voies d'eau, sont confrontés au devenir des sédiments et se trouvent démunis pour assurer une gestion à terre rigoureuse (réglementations, faisabilité technique, ...). La gestion durable à terre des sédiments constitue un intérêt indéniable de recherche comme en témoigne le nombre de projets réalisés et en cours dans ce domaine. On peut rappeler ici quelques travaux relatifs à la valorisation des sédiments (COLIN, 2003 ; BOUTOUIL, 2006; DUBOIS, 2006; LECLERC, 2006; LEMEE, 2006 ; BEHMANESH, 2008 ; DUAN, 2008 ; NGUYEN, 2008 ; LIMEIRA et al., 2009 ; SILITONGA et al., 2009 ; TRAN, 2009, LEVACHER 2010). Si l'on se réfère aux volumes de sédiments dragués en France de 50 à 60 millions de $\mathrm{m}^{3}$ (BOUTIN, 1999 ; BOUTOUIL, 2010), la gestion de ces volumes i.e. stockage, recyclage, valorisation, ne peut s'envisager qu'à une échelle industrielle. En effet, valoriser les gros volumes qui concernent les sédiments sains constitue une alternative au rejet en mer (clapage) ou au stockage à terre (casiers de remplissage, ballastières). C'est la problématique I du tableau 1.

Dès lors qu'une contamination des sédiments est avérée, les éliminer devient une contrainte environnementale et sociétale forte (AQUA et al., 2009, ENIAZ \& DAMIDOT, 2010). Cette élimination souhaitée comme définitive, passe par un recyclage complet après traitement de décontamination, c'est la problématique II du tableau 1.

La problématique I, de par le caractère renouvelable de la source en sédiments et ses volumes générés, est du point de vue économique i.e. coût du produit valorisé et valeur ajoutée, celle à privilégier. La problématique II concerne des actions plus ponctuelles (effet local, pollution par contaminant isolé) avec une série de prétraitements appropriés. Cependant la valorisation des sédiments et la recherche de filières de valorisation ont montré leurs limites et les difficultés se traduisent par les verrous scientifiques et/ou techniques suivants à lever :

- verrou I : déshydratation directe des sédiments marins et fluviaux à la sortie de la drague mécanique ou hydraulique, avec ou sans reprise du sédiment ;

- verrou II : stabilisation/solidification à base de liants et d'additifs et recherche de formulations robustes ;

- verrou III : manque de recherches constituant des voies nouvelles de traitement.

Tableau 1. Problèmatiques posées pour le devenir des sédiments.

\begin{tabular}{lllll}
\hline Problématique & Action & $\begin{array}{l}\text { Ressource en } \\
\text { sédiments }\end{array}$ & Volumes & $\begin{array}{l}\text { Etats des } \\
\text { sédiments }\end{array}$ \\
\hline Problématique & $\begin{array}{l}\text { Alternative au clapage et au } \\
\text { stockage }\end{array}$ & Renouvelable & Importants & $\begin{array}{l}\text { Sédiments } \\
\text { sains }\end{array}$ \\
\hline Iroblématique & $\begin{array}{l}\text { Restauration et maintien de } \\
\text { ports, fleuves et canaux propres }\end{array}$ & $\begin{array}{l}\text { Non } \\
\text { renouvelable }\end{array}$ & Réduits & $\begin{array}{l}\text { Sédiments } \\
\text { pollués }\end{array}$ \\
\hline II & & & \\
\hline
\end{tabular}




\section{XI ${ }^{\text {èmes }}$ Journées Nationales Génie Côtier - Génie Civil Les Sables d'Olonne, 22-25 juin 2010}

Le projet SEDIGATE (SEDIments - Gestion A TErre) a été élaboré pour lever ces verrous et apporter des réponses à un niveau industriel tout en considérant les coûts et l'approche " carbone » dans les procédés de conditionnement et de traitements. Il se décline en 3 projets conformément aux verrous à lever. SEDIGATE I, objet de cette communication, porte sur la déshydratation mécanisée in situ de sédiments fraîchement dragués ou mis en dépôts et la mise en conditionnement des sédiments déshydratés pour être transportés, traités et valorisés suivant différentes filières, tableau 2.

Tableau 2. Domaines possibles de valorisation.

\begin{tabular}{ll}
\hline Objectif & Application et domaine concerné \\
\hline $\begin{array}{l}\text { 1- Pelletabilité du produit en cours de } \\
\text { stabilisation/solidification. }\end{array}$ & $\begin{array}{l}\text { Transport et mise en décharge (matériau fin } \\
\text { contaminé), mise en dépôt pour stockage. }\end{array}$ \\
$\begin{array}{l}\text { cavités souterraines. } \\
\text { 3- Réalisation de remblai sans surcharge. }\end{array}$ & $\begin{array}{l}\text { Matériau de remplissage, de comblement. } \\
\text { Consolidation sous poids propre, zones } \\
\text { aménages. }\end{array}$ \\
4- Remblai avec surcharge. & $\begin{array}{l}\text { Matériau de terre-pleins et de plates-formes } \\
\text { industriels. }\end{array}$ \\
5- Valorisation en technique routière. & Matériau de sous-couches routières. \\
6- Valorisation en matériaux de construction. & Matériaux : briques, tuiles, ... \\
7- Valorisation en matériaux élaborés. & $\begin{array}{l}\text { Matériaux renforcés avec fibres : } \\
\text { récifs artificiels, matériaux du BTP. }\end{array}$ \\
8- Co-valorisation avec d'autres déchets. & Matériaux de construction et routes. \\
\hline
\end{tabular}

\section{Déshydratation naturelle des sédiments}

La déshydratation fait partie d'un ensemble de procédés appliqués au traitement des sédiments mais elle constitue une phase essentielle dans les processus de valorisation ou de stockage futur, voir figure 1. Sur cette figure, la configuration des postes de pré traitement permet de réduire le volume des matériaux par déshydratation et/ou par séparation granulaire. Cette chaîne de prétraitement comme dans le projet SEDIMARD 83 (SANNIER et al., 2009) peut être composée de deux cribles vibrants (tri des macro déchets supérieurs à $20 \mathrm{~mm}$, puis les particules entre 2 et $20 \mathrm{~mm}$ ), d'un hydrocyclone (séparation des sables, fraction $0,63 / 2 \mathrm{~mm}$ ), puis d'un décanteur (épaississement des fines). Les fines, selon les chaînes mises en oeuvre, sont envoyées soit sur un filtrepresse (action mécanique), soit directement en bioremédiation (SANNIER, 2008). Ainsi cette chaîne comprend 4 à 5 machines mises en série avec un fonctionnement en discontinu (filtre-presse). La déshydratation peut s'opérer au moindre coût par voie de séchage naturel, être mécanisée, réalisée par voie chimique et/ou thermique tout en notant que le coût va croître respectivement.

Le séchage naturel, pour une grande efficacité requiert d'importantes surfaces au sol, souvent indisponibles de la part des gestionnaires de ports et des voies d'eau. De plus, 
pour atteindre les teneurs en eau optimales pour valoriser les sédiments, le temps de séchage dépend des conditions de stockage en milieu abrité ou non, de l'épaisseur des couches, du remuage des couches, de la teneur en eau initiale, des caractéristiques hydrauliques des sédiments, (SANCHEZ \& LEVACHER, 2007 ; LEVACHER, 2008 ; LEVACHER \& SANCHEZ, 2010) et enfin, peut s'avérer long et incompatible avec des applications industrielles. Les données relatives au séchage de sédiments mis en dépôts par couche sur un lit drainant sont peu disponibles dans la littérature (DUBOIS, 2006). On peut citer le cas de l'égouttage d'un sédiment portuaire de Cherbourg effectué en laboratoire (DUAN, 2008), figure 2, l'expérience des sédiments compostés sur le site pilote de traitement de SEDIMARD 83 (SANNIER et al., 2009).

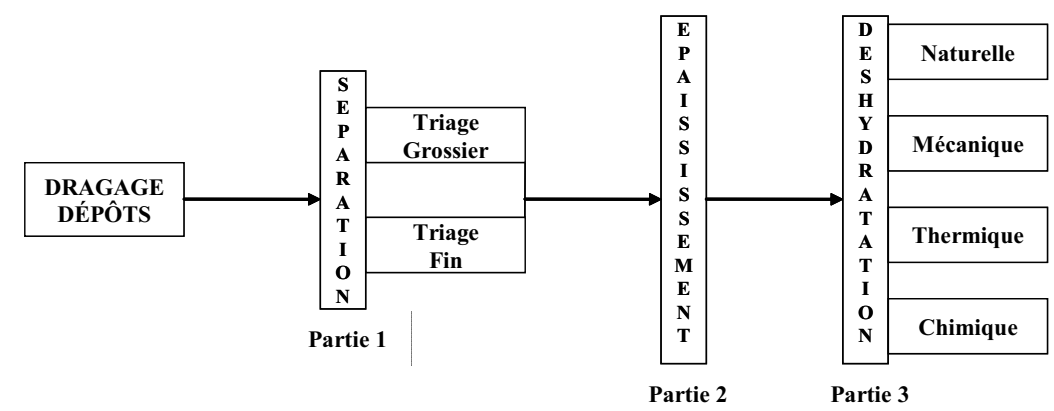

Figure 1. Cheminement des sédiments en début de traitement.

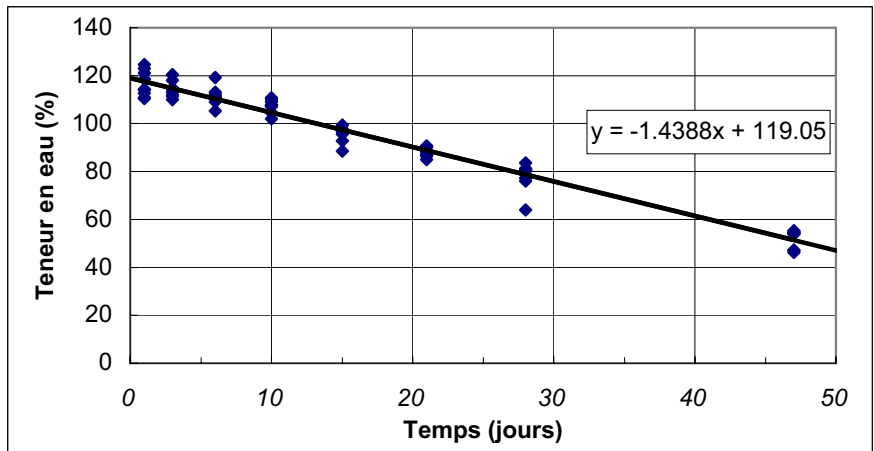

Figure 2. Relation teneur en eau-temps, cas d'un sédiment portuaire de Cherbourg.

\section{Contenu du projet SEDIGATE I}

\subsection{Le projet et la machine à déshydrater prototype}

Le projet SEDIGATE considère que le verrou scientifique -verrou I- est en amont de tout type de traitement de stabilisation/solidification qui nécessite des sédiments à teneur en eau faible (siccité élevée $>50 \%$ ). C'est une phase dite de conditionnement des sédiments pour en assurer le stockage, le transport et le traitement. Ce projet dénommé SEDIGATE I, a pour objet de déshydrater et de trier des sédiments récupérés en sortie de drague pour des débits inférieurs à $300 \mathrm{~m}^{3} / \mathrm{h}$ ou repris en zone tampon de stockage 


\section{XI $I^{\text {èes }}$ Journées Nationales Génie Côtier - Génie Civil}

Les Sables d'Olonne, 22-25 juin 2010

(casier, conteneur). Le procédé assure une séparation sables/fines particules. La siccité des boues (particules fines) en sortie de machine est convenable pour tout type de traitement de stabilisation/solidification ou tout transport. La durée du projet SEDIGATE I est de 3 ans sur la période 2010-2013. Pour atteindre cet objectif, la création d'une unité mobile de conditionnement UMC a été nécessaire. Tout d'abord un prototype à échelle réduite (figure $3 \mathrm{a}$, corps principal cylindrique de $2 \mathrm{~m}$ de diamètre, de $10 \mathrm{~m}$ de longueur, masse totale de $8 \mathrm{t}$ ), a été testé montrant l'efficacité du procédé mécanique retenu, séparant sables et fines (figure 3b) puis rejetant l'eau (figure 3c). Cette machine -seule-, de surcroît mécanisée et mobile, sépare les sables et graviers de la matrice fine et conditionne celle-ci à une siccité voisine de 25 à $30 \%$.

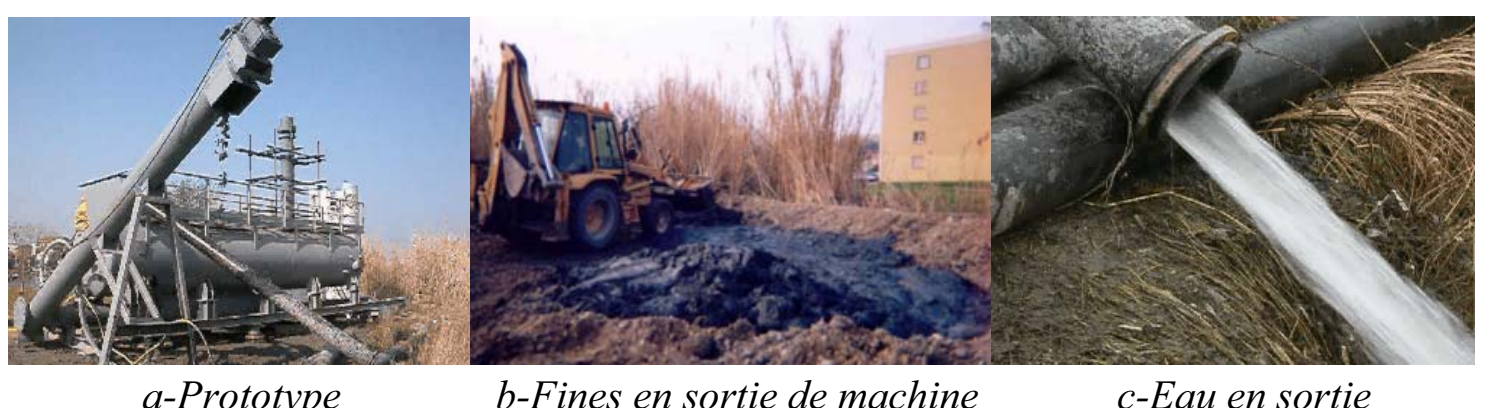

Figure 3. La machine à déshydrater : le prototype en action.

\subsection{L'unité mobile de conditionnement-UMC}

Les essais réalisés à l'aide du prototype jugés probants, ont permis d'envisager la construction d'une machine à déshydrater à échelle réelle. Elle est composée de l'équivalent de 3 conteneurs dont deux superposés B et C (voir figure 4a), aisément démontables et transportables par voie routière, fluviale ou maritime.

Les trois conteneurs sont connectés de manière à :

- assurer l'arrivée des sédiments et l'injection de floculants ainsi que l'ensemble de l'automatisation, conteneur A;

- séparer les sables et graviers des fines, conteneur B ;

- récupérer les sables et les fines, conteneur $\mathrm{C}$ et figure $4 \mathrm{~b}$.

La première machine à déshydrater dénommée unité mobile de conditionnement UMC est disponible et sera mise en opération en mai 2010 (ARD, site web). La capacité de la machine est de traiter des sédiments sains à une cadence allant de 150 à $300 \mathrm{~m}^{3} /$ heure et de viser une siccité pour les fines, supérieure à $25-30 \%$. Les atouts de cette machine par rapport à l'existant est (i) d'assurer le tri, la séparation, la déshydratation mécanisée en continu pour une capacité moyenne de $225 \mathrm{~m}^{3} /$ heure pour des sédiments en sortie de drague, repris en dépôts ou prélevés sur site, et (ii) d'être mobile du fait qu'on peut l'installer en poste à proximité des sédiments à déshydrater à savoir dans un grand port, 
dans un port de plaisance, sur berge près de canaux et même sur barge avec une emprise au sol équivalente à deux conteneurs.

Les premiers chantiers porteront sur la déshydratation de sédiments fluviaux et maritimes sains avec comme produits de sortie: l'eau (rejet sur le site de prélèvement), les matériaux grossiers (lavage éventuel et mise sur le marché de granulats) et les fines prêtes pour le transport, la transformation ou la valorisation sur site.

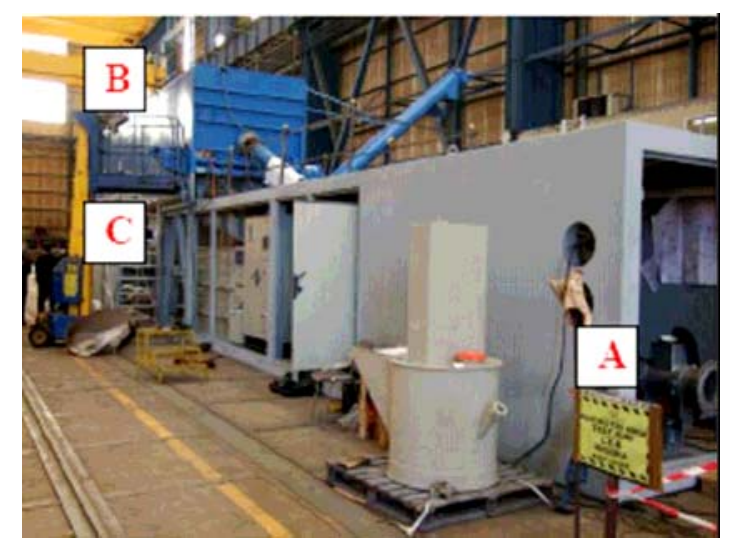

a-Machine en fin de construction

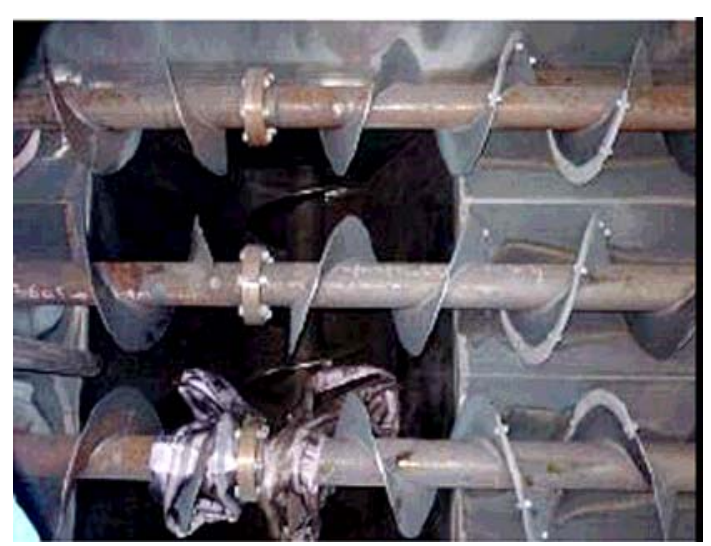

b-Raclage des sédiments fins

Figure 4. La machine à déshydrater : la machine en taille réelle.

\section{Les développements futurs autour de la machine à déshydrater}

Le projet SEDIGATE I consiste à confirmer le bon fonctionnement du procédé breveté par l'utilisation de la machine sur site puis d'établir les performances et les limites de l'unité mobile de conditionnement, UMC, qui seront évaluées à partir de l'analyse des paramètres suivants : qualité et nature du sédiment, débits entrée, dosage en floculants, siccité des sédiments en sortie, tris. L'application est prévue sur un sédiment fluvial puis marin, pour considérer la salinité au niveau des performances des floculants biodégradables.

Les premiers développements concernent ( $i$ ) l'amélioration de la siccité en sortie de machine au niveau du concept d'une unité de pressage (UP), (ii) le confinement des fines déshydratées pour la confection de géo enveloppes géosynthétiques ou équivalents (unité de pressage et de confinement, UPC), (iii) l'adaptation de l'UMC sur site à la réception de sédiments contaminés (unité pour sédiments pollués, USP) qui intégrera le traitement en masse (figure 5) des sédiments déshydratés et le traitement de l'eau.

\section{La recherche appliquée en appui}

Le caractère très technique du projet SEDIGATE I en fait un projet industriel innovant très en amont de la valorisation. Il convient donc de développer à la suite de l'action de déshydratation des recherches plus fondamentales dans le cadre de la valorisation. Ce sont les projets qui correspondent aux verrous II et III décrits en introduction. 

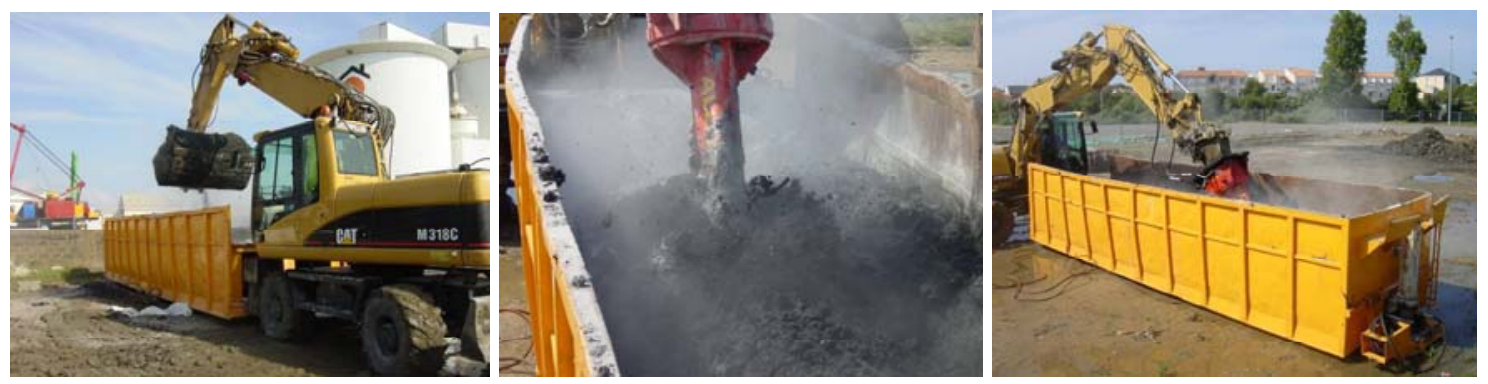

Figure 5. Traitement en masse de sédiments d'après la société ALLU France (site web).

\subsection{Le projet SEDIGATE II}

Le projet dénommé SEDIGATE II consiste à proposer des formulations robustes, fiables et simples à mettre en œuvre du point de vue industriel. Les constituants de ces formulations sont à la base soit des liants hydrauliques classiques soit des liants pouzzolaniques et ajouts d'activation (ARD, site web ; LEMEE, 2006 ; LIANG, 2008 ; OBANA et al., 2009 ; SILITONGA et al., 2008 ; SILITONGA et al., 2010). La durée de ce projet est de 4 ans sur 2011-2014 dont les résultats concernent essentiellement qui pourront faire l'objet de guides méthodologiques pour chaque filière envisagée en construction piétonne et routière. L'aboutissement industriel est la création d'une unité de mélanges sédiments-liants qui viendra en complémentarité de l’UMC.

\subsection{Le projet SEDIGATE III}

Le projet référencé SEDIGATE III est prévu sur une période plus longue de 5 ans (2011-2015) de par son caractère fondamental et innovant. C'est une action de recherche et développement dont l'objet porte sur la recherche de voies nouvelles de valorisation en considérant les principes et concepts suivants :

- une covalorisation de sédiments et de déchets, l'idée force est de faire chuter le prix final du produit élaboré et d'éliminer un autre déchet. Les mélanges avec des fines ou sables de carrière non vendables sont envisagés comme avec des déchets : fibres végétales, SEDIFIBRES ${ }^{\circledR}$, déchets coquilliers à forte teneur de $\mathrm{CaCO}_{3}$ ou à forte teneur en silice, autres déchets fibreux (LEVACHER \& LIANG, 2009).

- Une banque de données sur les sédiments depuis leur prélèvement (données géotechniques) au traitement (formulations appliquées) et application (données relatives à l'application). Cette banque de données peut être associée à la sédimentothèque, idée innovante du projet SEDIMARD 83 (AQUA et al., 2009 ; SANNIER et al., 2009; SEBY et al., 2009) qui a recueilli l'ensemble des échantillons des sédiments prélevés et traités dans le cadre de ce projet.

\section{Conclusions}

La déshydratation des sédiments est un des points clés de toute valorisation. Pour réaliser cette action, on met en place successivement plusieurs machines qui opèrent 
tris, séparation et déshydratation. Le projet SEDIGATE I propose à l'aide d'une seule machine mobile et automatisée de déshydrater des sédiments fraîchement dragués ou mis en dépôts. L'optimisation des paramètres d'utilisation de cette machine fait partie du premier chantier appliqué à un sédiment fluvial. Par la suite, les projets de recherche et de développement SEDIGATE II et III viennent assurer du point de vue industriel, les traitements en vue de la valorisation de sédiments sains dans un premier temps, puis de sédiments peu contaminés.

\section{Remerciements}

Les auteurs remercient la Région Basse Normandie à travers Normandie Incubation d'avoir labellisé le projet SEDIGATE ${ }^{\circledR}$ I.

\section{Références}

ALLU (site web) http://www.allu.net

AQUA J.-L., BOISSERY P., ALZIEU C. (2009). Un programme opérationnel d'identification des modes de gestion durable des sédiments marins : le projet SEDIMARD 83. Revue Paralia, $\mathrm{n}^{\circ}$ 2, pp s1.1-s1.12. doi:10.5150/revue-paralia.2009.s01

ARD (site web). ARD, la référence des sols stabilisés naturels. http://www.ardnormandie.fr.

BEHMANESH J. (2008). Etude de la durabilité d'un sédiment traité au ciment et additifs. Thèse de Doctorat, Université de Caen, 215 p.

BOUTIN R. (1999). Amélioration des connaissances sur le comportement des rejets en mer de produits de dragage de type vase. Thèse INSA, Institut National des Sciences Appliquées de Lyon, $527 \mathrm{p}$.

BOUTOUIL M. (2006). Traitement des vases de dragage par solidification/stabilisation à base de ciment et additifs. Habilitation à Diriger des Recherches, Université de Caen, 101 p.

BOUTOUIL M. (2010). Présentation du projet SETARMS. II ${ }^{\text {nd }}$ International Symposium on Sediments Management, I2SM, Casablanca, 11-13 may 2010, in press.

COLIN D. (2003). Valorisation de sédiments fins de dragage en technique routière. Thèse de Doctorat, Université de Caen, 180 p.

DUAN Z. (2008). Caractérisation, stabilisation et solidification de sédiments marins. Thèse de Doctorat, Université de Caen, $144 \mathrm{p}$.

DUBOIS V. (2006). Etude du comportement physico-mécanique et caractérisation environnementale des sédiments marins. Valorisation en technique routière. Thèse de Doctorat, Ecole Nationale Supérieure des Mines de Douai, 190 p. + annexes 95 p.

ENIAZ E., DAMIDOT D. (2010). Sédimatériaux. II $^{\text {nd }}$ International Symposium on Sediments Management, I2SM, Casablanca, 11-13 may 2010, in press.

LECLERC M. (2006). Valorisation de la tangue, recherche de solutions et analyse technico-économique. Mémoire de stage ENTPE, DDE Manche, 98 p. 
LEMEE F. (2006). Traitement par alcali activation de sédiments fins marins non contaminés et à faible teneur en eau - Mise au point d'un procédé de stabilisation. Thèse de doctorat, Université de Caen Basse-Normandie, $187 \mathrm{p}$.

LEVACHER D. (2008). Caractérisation géotechnique des sédiments : l'exemple des ports méditerranéens. Colloque La gestion durable des sédiments, une problématique, des spécificités méditerranéennes, Monaco, 28-29 octobre 2008, version CD- Ed. RAMOGE Monaco.

LEVACHER D. (2010). Some aspects on the sediment valorization: characterization, treatment, coast, recent developments. $\mathrm{II}^{\mathrm{nd}}$ International Symposium on Sediments Management, I2SM, Casablanca, 11-13 may 2010, in press.

LEVACHER D., LIANG Y. (2009). Comparaison de l'efficacité de différentes additions dans des procédés de stabilisation/solidification pour sédiments marins. 2èmes Journées d'Etudes sur la recherche en Environnement et Développement durable - 2èmes JEREDD-09-3-4 Juin 2009, Oran, version CD.

LEVACHER D., SANCHEZ M. (2010). Caractérisation de sédiments marins pour une mise en dépôt à terre et en remblai, European Journal of Environmental and Civil Engineering, EJECE, numéro spécial « Sédiments »,12 pages, in press.

LIANG Y. (2008). Traitement de sédiments marins : amélioration de la résistance par des procédés de stabilisation/solidification par voie chimique et liants hydrauliques. Rapport Master 2 Recherche Génie Côtier, Université de Caen, 53 p.

LIMEIRA J., AGUlLO L., ETXEBERRIA M. (2009). Dredged marine sand in concrete: An experimental section of a harbor pavement. Construction and Building Materials, doi:10.1016/j.conbuildmat.2009.12.011

NGUYEN T.B. (2008). Valorisation des sédiments de dragage traités par le procédé Novosol dans des matériaux d'assises de chaussée. Comportement mécanique et environnemental. Thèse de Doctorat, Institut National des Sciences Appliquées de Toulouse, $244 \mathrm{p}$.

OBANA M., LEVACHER D., DHERVILLY P. (2009). Comportement mécanique de la tangue traitée avec un liant et un produit chimique réactif. 1ère Conférence Méditerranéenne en Côtière et Maritime, CM2, Hammamet, Tunisie, pp 151-154. doi:10.5150/cmcm.2009.038-1

SANCHEZ M., LEVACHER D. (2007). The influence of particle size of the dispersed mineral fraction on the settlement of marine and estuarine muds. Geo-marine Letters, Volume 27, $\mathrm{n}^{\circ}$ 5, Octobre, pp 303-313. doi:10.1007/s00367-007-0053-7

SANNIER L., (2008) Limites et performances des caractéristiques mécaniques et environnementales de sédiments marins contaminés traités par liant hydraulique. Application au pilote SEDI.MAR.D. 83, Mémoire de Master de Recherche, Université de Caen, 106 p. 
SANNIER L., LEVACHER D., JOURDAN M. (2009), Approche économique et validation de méthodes de traitements aux liants hydrauliques de sédiments marins contaminés. Revue Paralia, ${ }^{\circ}$ 2, pp s2.1-s2.15. doi:10.5150/revue-paralia.2009.s02

SEBY F., BENOIT-BONNEMASON C., TESSIER E., ALZIEU C., AQUA J.-L., SANNIER L., DONARD O.F.X. (2009). Etude de l'évolution des formes chimiques des métaux dans des sédiments marins dragués stockés à terre. Revue Paralia, $\mathrm{n}^{\circ} 2$, pp s3.1-s3.12. doi:10.5150/revue-paralia.2009.s03

SILITONGA E., SHRIVASTAVA A., LEVACHER D. (2008). Influence of fly ash addition on the mechanical properties of treated dredged material. 1st International Symposium on Sediment Management, I2SMLille, July 9-11th , 2008, pp 243-252.

SILITONGA E., LEVACHER D., MEZAZIGH S. (2009). Effects of the use of fly ash as a binder on the mechanical behaviour of treated dredged sediments. Environmental Technology, Volume 30, Issue 8, pp 799-807. doi:10.1080/09593330902990089

SILITONGA E., LEVACHER D., MEZAZIGH S. (2010). Utilization of fly ash for stabilization of marine dredged sediments. European Journal of Environmental and Civil Engineering, volume14, $\mathrm{n}^{\circ}$ 2, pp 253-265. doi:10.3166/ejece.14.253-265

TRAN N.T. (2009). Valorisation de sédiments marins et fluviaux en technique routière. Thèse de Doctorat, Ecole Nationale Supérieure des Mines de Douai, 187 p. 